\title{
A SPECIAL CLASS OF MOUFANG LOOPS
}

\author{
HALA ORLIK-PFLUGFELDER
}

Abstract. Loops satisfying the identical relation $(x y)(z x)$ $=[x(y z)] x$ are known as Moufang loops. In the present paper considered is a class of loops satisfying the identical relation

(1)

$$
(x y)\left(z x^{\lambda}\right)=[x(y z)] x^{\lambda},
$$

where $x^{\lambda}$ is the image of $x$ under some mapping $\lambda$ of the loop into itself. Loops satisfying (1) are called $M$-loops.

If $\lambda: x \rightarrow x^{k}, k$ an integer, (1) is called an $M_{k}$-law. It is shown that every $M$-loop is Moufang, and every $x^{\delta}=x^{-1} \cdot x^{\lambda}$ belongs to the nucleus.

Furthermore, if a loop satisfies (1) so do all its loop-isotopes.

I. Introduction. In a commutative loop the mapping $x \rightarrow x^{3}$ is a centralizing endomorphism. The question arose whether one could generalize this fact and determine a class of loops with a centralizing endomorphism $x \rightarrow x^{n}$ for an arbitrary integer $n$. The first idea that comes to mind is to ask, even in the noncommutative case, how Moufang loops generalize when the Moufang law $(x y)(z x)=(x(y z)) x$ is replaced by a seemingly more general one

$$
(x y)\left(z x^{k}\right)=(x(y z)) x^{k}, \quad k>1 .
$$

In this paper we answer this question obtaining a number of results, all containing as particular case, for $k=1$, corresponding ones for Moufang loops. In fact, we go even further, replacing the mapping $x \rightarrow x^{n}$ by any single-valued mapping $\lambda$ of the loop into itself. ${ }^{1}$

In a preliminary report (Notices Amer. Math. Soc. 16 (1969), Abstract 664-753) some of these results were stated as generalizations of corresponding ones for Moufang loops. Somewhat surprisingly, it turns out that if (1) holds for any integer $k$ it also holds for $k=1$ (see Theorem 1). It follows that the structure induced on a loop by (1) for $k>1$ is not more general than that of Moufang loops, but, on the contrary, (1) defines a restriction of them.

II. Basic definitions and properties of $M$-loops. Let $G$ be a loop and $\lambda, \delta$ two single-valued mappings of $G$ into itself such that $\lambda=1+\delta$ in the sense that

Received by the editors March 31, 1969 and, in revised form, January 16, 1970.

AMS 1969 subject classifications. Primary 2095.

Key words and phrases. Moufang loop, inverse property loop (I.P. loop), di-associative property, neoring, $M$-loop, $M_{k}$-loop, strictly Moufang autotopism, pseudoautomorphism with companion $C$, loop-isotopy.

1 The author wishes to thank Professor R. H. Bruck for this valuable suggestion which improved the paper considerably. 


$$
x^{\lambda}=x \cdot x^{\delta} \quad \text { for every } x \text { in } G .
$$

THEOREM 1. If $G$ satisfies the identical relation

$$
(x y)\left(z x^{\lambda}\right)=(x(y z)) x^{\lambda}
$$

then $G$ is Moufang and $G \delta \subset N$, where $N$ is the nucleus of $G$. Conversely, if $G$ is Moufang with nucleus $N$, the set $D$ of all maps $\delta: G \rightarrow N$ is an associative left neoring, and (3) is an identical relation in $G$ for every $\lambda$ defined by (2) for every $\delta \in D$.

To prove this theorem we will need two lemmas:

LEMma 1. If $G$ satisfies (3) then $L(x) R\left(x^{\lambda}\right)=R\left(x^{\lambda}\right) L(x)$, where $L(x), R(x)$ are left and right multiplications by $x$, respectively.

The proof follows from (3) with $y=1$.

Lemma 2. If a loop $G$ satisfies (3) then $G$ has the inverse property.

Proof. We define $x^{-1}$ by $x x^{-1}=1$. Using (3) with $y=x^{-1}$ we have $\left(x\left(x^{-1} z\right)\right) x^{\lambda}=\left(x x^{-1}\right)\left(z x^{\lambda}\right)=z x^{\lambda}$, or

$$
x\left(x^{-1} z\right)=z,
$$

which is the left inverse property. From (4) with $z=x: x\left(x^{-1} x\right)=x$, $x^{-1} x=1$ and

$$
\left(x^{-1}\right)^{-1}=x
$$

Setting $z=y^{-1} x^{-1}$ in (3) we have $(x y)\left(\left(y^{-1} x^{-1}\right) x^{\lambda}\right)=\left(x\left(y\left(y^{-1} x^{-1}\right)\right)\right) x^{\lambda}$ $=x^{\lambda}$ and

$$
(x y)^{-1}=y^{-1} x^{-1}
$$

To prove the right inverse property we choose $y^{-1}=z x^{\lambda}, x^{\lambda}=z^{-1} y^{-1}$ $=(y z)^{-1}$, and substitute it into (3):

$$
(x y) y^{-1}=(x y)\left(z x^{\lambda}\right)=x\left((y z) x^{\lambda}\right)=x\left((y z)(y z)^{-1}\right)=x .
$$

This completes the proof of Lemma 2.

We can now prove Theorem 1:

(i) An autotopism of a loop $G$ is a triple $(U, V, W)$ of permutations of $L$ such that $y U \cdot z V=(y z) W$ for all $x, y, z \in G$. If $(U, V, W)$ and $\left(U^{\prime}, V^{\prime}, W^{\prime}\right)$ are autotopisms of $G$ then the following triples are also autotopisms of $G$ : (UU', $\left.V V^{\prime}, W W^{\prime}\right),(W, J V J, U)$ and $(J U J, W, V)$, where $J$ is the inverse mapping [1, p. 112]. If (3) holds in $G$, then the following triples are autotopisms:

$$
\begin{gathered}
A(x)=\left(L(x), R\left(x^{\lambda}\right), L(x) R\left(x^{\lambda}\right)\right), B(x)=\left(R\left(x^{-1}\right), L(x) R\left(x^{\lambda}\right), R\left(x^{\lambda}\right)\right), \\
A(x) B(x)^{-1}=\left(L(x) R(x), L(x)^{-1}, L(x)\right), \quad C(x)=(L(x), R(x), L(x) R(x)) .
\end{gathered}
$$


Since $C(x)$ is an autotopism of $G, G$ is Moufang.

(ii) Being Moufang, $G$ is diassociative. Using diassociativity of $G$ and (3) we have an autotopism $D(x)=A(x) C\left(x^{-1}\right)=\left(I, R\left(x^{\delta}\right)\right.$, $R\left(x^{\delta}\right)$ ), or $y \cdot z x^{\delta}=(y z) x^{\delta}$, which implies that $x^{\delta} \in N$ for all $x \in G$. This proves the first part of the theorem.

(iii) To prove the converse we consider a Moufang loop $G$ and the set $D=\{\delta\}$ of all mappings of $G$ into $N$. It is easy to show that $D$ is an associative left neoring with respect to operations $(\oplus)$ and $(0)$ defined as follows:

$$
\begin{aligned}
x\left(\delta_{1} \oplus \delta_{2}\right) & =x \delta_{1} \cdot x \delta_{2}, \quad x \in G, \\
x\left(\delta_{1} \circ \delta_{2}\right) & =\left(x \delta_{1}\right) \delta_{2} .
\end{aligned}
$$

(A left neoring is a system $(R,+, \cdot)$ with two binary operations such that $(R,+)$ is a loop with identity $0, R$ is closed under $(\cdot), x \cdot 0=0$ and $x(y+z)=x y+x z$ for all $x, y, z \in G[3$, p. 50].) In our case $(D, \oplus)$ is a group, not necessarily Abelian, the identity being the trivial mapping $\delta_{0}: x \rightarrow e$. $(D, 0)$ is closed and $\delta_{i} \circ \delta_{0}=\delta_{0}$ for every $\delta_{i}$. The left distributive law also holds. The right distributive law holds in $(D, \oplus, 0)$ only if every $\delta \in D$ is an endomorphism.

(iv) Finally, from the Moufang law with $x^{\delta} \in N$, (3) follows:

$$
(x y)(z x) x^{\delta}=((x(y z)) x) x^{\delta}, \quad(x y)\left(z x^{\lambda}\right)=(x(y z)) x^{\lambda} .
$$

For the sake of convenience we wish to introduce the following definitions. A loop $G$ will be called an $M$-loop if it satisfies (3) for some single-valued mapping $\lambda$ of $G$ into itself. The identical relation (3) will be called the $M_{\lambda}$-law. Every Moufang loop is an $M$-loop since it satisfies (3) for $\lambda$ the identical mapping. If a loop $G$ satisfies the $M_{\lambda^{-}}$ law for $\lambda$ being the trivial mapping $x \rightarrow e, G$ is a group. Of a special interest are loops where $\lambda$ is a power-mapping $x \rightarrow x^{k}$ for an integer $k$. The identical relation (3) will in this case be called an $M_{k}$-law. If $G$ is a loop, and $k$ is the smallest positive integer $>1$ such that the $M_{k}$-law holds in $G$ but $x^{k-1}=1$ does not hold, the loop $G$ will be called an $M_{k}$-loop. A loop which is an $M$-loop but not an $M_{k}$-loop will be called strictly Moufang.

The following properties of $M$-loops are corollaries of Theorem 1:

COROLlaRY 1. In an $M_{k}$-loop, the $x^{(n(k-1)+3)}$ is a companion of some pseudo-automorphism upon $G$, for every $x \in G$.

This follows from Theorem 1 and Bruck's theorem that every element of $N G^{3}$ of a Moufang loop $G$ is a companion [2, p. 59].

CoRollary 2. If a commutative loop $G$ satisfies an $M_{k}$-law for $k \neq 1$ ( $\bmod 3)$ then $G$ is an Abelian group. 
Proof. If $G$ satisfies an $M_{k}$-law, then $x^{k-1} \in N$ for every $x \in G$. On the other hand, in a commutative Moufang loop $x^{3} \in N$. If $(k-1,3)$ $=1$, or $k \neq 1(\bmod 3)$, every $x \in N$ and $G$ is a group.

Two loops $(G, \cdot)$ and $(G, 0)$ are called isotopic if there exists a triple of permutations $(\alpha, \beta, \gamma)$ of $G$ such that $x \alpha \cdot y \beta=(x \circ y) \gamma$. If $\gamma=1,(G, \oplus)$ is called a principal isotope of $(G, \cdot)$. A property $p$ of a loop is called a universal property if it is invariant under loop-isotopy.

THEOREM 2. An $M_{\lambda}$-law is a universal property of loops.

Proof. Let the loops $(G, \cdot)$ and $(G, \circ)$ be isotopic and $(G, \cdot)$ satisfy an $M_{\lambda}$-law. We have to show that the $M_{\lambda}$-law holds also in $(G, 0)$. Since the Moufang property is universal for loops, $(G, 0)$ is Moufang. Without loss of generality, we can assume that $(G, 0)$ is a principal isotope of $(G, \cdot)$ and $x \circ y=x f \cdot f^{-1} y\left[1\right.$, p. 129]. If $x^{\delta}$ belongs to the nucleus of $(G, \cdot)$ it also belongs to the nucleus of $(G, 0)$ :

$$
\begin{aligned}
& (y \circ z) \circ x^{\delta}=\left(\left(y f \cdot f^{-1} z\right) f\right)\left(f^{-1} x^{\delta}\right)=\left(y f \cdot f^{-1} z\right) x^{\delta}, \\
& y \circ\left(z \circ x^{\delta}\right)=(y f)\left(f^{-1}\left(z f \cdot f^{-1} x^{\delta}\right)\right)=(y f)\left(f^{-1} \cdot z x^{\delta}\right)=\left(y f \cdot f^{-1} z\right) x^{\delta},
\end{aligned}
$$

and $(y \circ z) \circ x^{\delta}=y \circ\left(z \circ x^{\delta}\right)$. Now, $x^{\delta} \in N(G, \circ)$ together with the Moufang law implies the $M_{\lambda}$-law for $(G, 0)$.

\section{REFERENCES}

1. R. H. Bruck, A survey of binary system, Ergebnisse der Mathematik und ihrer Grenzgebiete, Heft 20, Springer-Verlag, Berlin and New York, 1958. MR 20 \#76.

2. - Some theorems on Moufang loops, Math. Z. 73 (1960), 59-78. MR 23 \#A3192.

3. - Analogues of the ring of rational integers, Proc. Amer. Math. Soc. 6 (1955), 50-58. MR 16, 1083

Temple University, Philadelphia, Pennsylvania 19122 